\title{
Galactic plane structure in hard X-rays
}

\author{
A. Lutovinov ${ }^{1}$, M.Revnivtsev ${ }^{2,1}$ and R.Krivonos ${ }^{2,1}$ \\ ${ }^{1}$ Space Research Institute, Profsoyuznaya str. 84/32, Moscow, 117997, Russia \\ ${ }^{2}$ Max-Plank Institute fur Astrophysics, Karl-Schwarzschild str. 1, Garching, Germany
}

\begin{abstract}
We study the structure of the Galaxy in the hard X-ray energy band (¿20 keV) using data from the INTEGRAL observatory. The increased sensitivity of the survey and the very deep observations performed during six years of the observatory operation allow us to detect about a hundred new sources. This significantly enlarges the sample of hard X-ray sources in the Galactic disk and bulge in a comparison with the previous studies.
\end{abstract}

We are continuing studies of the Galaxy structure in hard X-rays ( $>20 \mathrm{keV})$ using data from the INTEGRAL observatory. Observations and discoveries of a large number X-ray sources during more than 6 years of its operation in orbit have allowed us to improve significantly our knowledge about different populations of X-ray binaries, their properties and spatial distribution (Lutovinov et al. 2005, 2006, 2008; Krivonos et al. 2007).

At the moment (August, 2009) the total number of confidently detected sources with INTEGRAL amounts to 480, 80 of which are high-mass X-ray binaries (HMXB) and 92 are low-mass X-ray binaries (LMXB). We constructed the distribution of hard Xray binaries in the Galaxy and found that they are concentrated towards the Galactic plane; however HMXBs and LMXBs have different vertical scale heights and spatial distribution along the plane, reflecting the age of stellar companions of these sources and their evolution. HMXBs are mostly located in spiral arms as a young galactic population, whilst LMXBs are concentrated to the Galactic Centre. It is worthy to note that the current INTEGRAL exposure and sensitivity reached in the Galactic plane allows us to detect hard X-ray sources with the luminosity of $L_{X}>10^{35.5} \mathrm{erg} \mathrm{s}^{-1}$ practically throughout the entire Galaxy.

We also demonstrated that the spatial distribution of persistent LMXBs in the Galactic Centre/Galactic bulge region is consistent with a model of stellar mass distribution that includes the nuclear stellar disk component in the innermost degree of the Galaxy. The spatial distribution of transient LMXBs detected in the Galactic Centre region indicates an increased fraction of transient sources in the innermost degree of the Galaxy with respect to outer regions (Revnivtsev et al. 2008).

\section{Acknowledgements}

This work is supported by RFBR grant number 07-02-01051.

\section{References}

Lutovinov, A., Revnivtsev, M., Gilfanov, M., et al., 2005, A\&A 444, 821

Lutovinov, A., Revnivtsev, M., Gilfanov, M., \& Sunyaev, R., 2006, IAUS 230, 340 (Eds. E.J.A. Meurs, G. Fabbiano; CUP)

Lutovinov, A., Revnivtsev, M., Gilfanov, M., \& Sunyaev, R., 2008, Proceedings of the 6th INTEGRAL Workshop, 2007, ESA SP-622, p.241 (Eds. S.Grebenev, R.Sunyaev, C.Winkler)

Krivonos, R., Revnivtsev, M., Lutovinov, A., et al., 2007, A\&A 475, 775

Revnivtsev, M., Lutovinov, A., Churazov E., et al., 2008, A\&A 491, 209 